\title{
Syndrome sérotoninergique - Approche analytique en Toxicologie Hospitalière
}

\section{Serotonin syndrome - Analytical procedures in emergency toxicology}

Marie-France KERGUERIS*(1), Vincent DANEL ${ }^{(2)}$

(1) Laboratoire de Pharmacologie Clinique, Toxicologie, C.H.U. - 44093 NANTES Cedex 1 (2) Unité de Toxicologie Clinique, C.H.U. - BP 217 - 38043 GRENOBLE Cedex 9

* Auteur à qui adresser la correspondance : Marie-France KERGUERIS, Laboratoire de Pharmacologie Clinique, C.H.U. - 44093 NANTES Cedex 1 - Tel : 0240084095 - Fax : 0240083996

(Reçu le 3 février 2002 ; accepté le 26 février 2002)

\section{RÉSUMÉ}

Les inhibiteurs de la recapture de la sérotonine (IRS), les inhibiteurs de la recapture de la sérotonine et de la noradrénaline (IRSNA) et les inhibiteurs de la monoamine oxydase (IMAO) sont des antidépresseurs agissant sur le système sérotoninergique. Seuls ou le plus souvent associés à d'autres molécules intervenant sur cette même neurotransmission ils peuvent donner lieu à un syndrome sérotoninergique (SS) potentiellement grave. Après un rappel des aspects cliniques et thérapeutiques de ce syndrome et des données pharmacocinétiques des IRS et IRSNA, classe d'antidépresseurs récente fréquemment impliquée, nous réalisons une revue des différentes méthodes permettant l'identification et la mesure des substances susceptibles d'agir sur la biodisponibilité de la sérotonine au niveau du système nerveux central. La diversité des molécules recherchées nécessite l'utilisation de méthodes de «screening» large utilisant à ce jour la CLHP-barrette de diodes oulet la CPG$S M$ avec une sensibilité permettant si possible la détection à des taux thérapeutiques. Des méthodes de dosage chromatographique appliquant les mêmes conditions analytiques à l'ensemble des molécules de la famille IRS-IRSNA sont intéressantes dans le suivi des intoxications par ces molécules.

\section{MOTS-CLÉS}

Syndrome sérotoninergique, toxicologie d'urgence.

\section{SUMMARY}

Selective serotonin reuptake inhibitors (SSRI), serotonin noradrénaline reuptake inhibitors (SNRI) and monoamine oxydase inhibitors (MAOI) are antidepressants acting on serotonine system. Alone or more often, in each other combination or with others serotonergic substances, they may cause a "serotonin syndrome» (SS) potentially fatal. First, a reminder of clinical and therapeutic aspects of the serotonin syndrome, and pharmacokinetic parameters of SSRI and SNRI, new antidepressant class frequently involved in SS, is described. Secondly, we review the different identification and quantification methods of the drugs that lead to an excess of serotonin availability in the CNS. The diversity of the searched substances requires large screening methods using today HPLC-DAD orland GC-MS with a sensitivity allowing, if possible, therapeutic levels detection. Some chromatographic methods for the simultaneous determination of ISSRI-SNRI class described in recent years may be useful in drug monitoring and clinical toxicology.

\section{KEY-WORDS}

Serotonin sydrome, emergency toxicology. 


\section{Introduction}

Plusieurs classes d'antidépresseurs, dont le mécanisme d'action affecte différents systèmes de neurotransmetteurs, sont disponibles à ce jour. Parmi ces antidépresseurs, une classe récente de plus en plus prescrite regroupant les inhibiteurs de la recapture de la sérotonine (IRS) et une classe plus ancienne les IMAO (inhibiteurs de la monoamine oxydase), agissent sur le système sérotoninergique. Seuls ou le plus fréquemment associés à d'autres molécules intervenant sur cette même neurotransmission, ils peuvent donner lieu à un syndrome sérotoninergique potentiellement grave. Nous nous proposons de discuter la démarche analytique de toxicologie d'urgence dans ce type de situation.

\section{Le Syndrome sérotoniner- gique}

De description complète assez récente (Tableau I), le syndrome sérotoninergique (SS) rend probablement compte de nombreuses situations de syndromes hyperthermiques d'origine médicamenteuse toxique mal étiquetés auparavant $(1,2,3,4)$. Il correspond à une stimulation excessive et toxique de la transmission sérotoninergique par un excès de sérotonine au niveau des neurones cérébraux.

Tableau I : Syndrome sérotoninergique : critères diagnostiques d'après Sternbach (1).

Anamnèse
L'apparition du syndrome coïncide avec l'introduction ou
l'augmentation des doses d'un agent pro-sérotoninergique
et d'au moins 3 des signes cliniques suivants.
Signes cliniques
Confusion ou hypomanie, agitation, myoclonies, hyperré-
flexie ostéo-tendineuse, sueurs profuses, frissons, trem-
blements, diarrhée, incoordination, hyperthermie.
Diagnostic différentiel
Après élimination des principaux diagnostics différentiels,
d'une hyperthermie en particulier, le syndrome est surve-
nu sans introduction ou modification récente de la posolo-
gie d'un neuroleptique.

\section{Circonstances d'apparition}

Le SS peut être provoqué par des médicaments augmentant les quantités de sérotonine au niveau des récepteurs centraux par action sur la synthèse, la recapture neuronale ou la libération de la sérotonine. Les interactions médicamenteuses représentent la cause la plus fréquente de survenue de ce syndrome. D'une façon générale l'association responsable comporte une molécule diminuant le catabolisme de la sérotonine (comme un IMAO) et une molécule augmentant sa production (tryptophane) ou diminuant sa recapture (ISRS, antidépresseurs tricycliques par exemple). Les classes pharmacologiques augmentant la biodisponibilité de la sérotonine au niveau du système nerveux central sont indiquées dans le Tableau II.

Parmi les interactions les mieux documentées, on peut citer $(1,2,3,4)$ :

- IMAO ou IRS et L-tryptophane.

- IMAO non sélectifs et ISRS. Compte tenu de la longue demi-vie d'élimination de certaines de ces substances, le syndrome peut survenir lors de l'introduction d'un médicament plusieurs semaines après l'arrêt du précédent. Cette association est sans doute la plus fréquemment rapportée.

- IMAO sélectifs ou non avec la clomipramine, la péthidine, le dextrométhorphane.

- IMAO-B (sélégiline utilisée dans le traitement de la maladie de Parkinson) avec des ISRS ou des antidépresseurs tricycliques.

- Le moclobémide a été rendu responsable de syndromes sévères lors d'intoxications poly-médicamenteuses comprenant aussi de la fluoxétine, de la clomipramine, ou de l'amitriptyline (4). Ainsi, un syndrome sérotoninergique peut survenir après une intoxication aiguë associant moclobémide et ISRS ou moclobémide et antidépresseurs tricycliques. Il n'y a pas nécessairement de relations entre les doses ingérées et la gravité du syndrome.

Des syndromes sérotoninergiques ont été rapportés après des surdosages par sertraline, fluoxétine (4), venlafaxine (5), IMAO non sélectifs (4), ISRS ou IMAO associés avec le lithium (le lithium facilitant la transmission sérotoninergique) $(6,7,8)$, les morphiniques dont plus récemment le Tramadol (9), et les triptans (10).

Tableau II : Classes pharmacologiques augmentant la biodisponibilité de la sérotonine au niveau du système nerveux central.

- Précurseur de la sérotonine : tryptophane.

- Inhibiteurs de la monoamine oxydase : non sélectifs et sélectifs.

Inhibiteurs de la recapture de la sérotonine : IRS et IRSNA, voir tableau III.

- Antidépresseurs tricycliques pro-sérotoninergiques : clomipramine, amitryptyline.

- Agonistes sérotoninergiques : triptans, dérivés de l'ergot, buspirone.

- Substances augmentant la libération de sérotonine : MDMA, 4-MTA.

Divers autres : Lithium, dérivés opiacés, cocaïne. 


\section{Clinique}

La forme modérée, la plus fréquente, peut se manifester par une modification de l'état mental, une agitation, des tremblements, des myoclonies, des sueurs, des frissons. Dans son expression la plus frustre, cette forme peut passer inaperçue si le diagnostic n'est pas évoqué (4).

La forme grave associe des troubles de conscience, des manifestations de dysautonomie neurovégétative, et des symptômes neuro-musculaires. Les examens de sang peuvent montrer une hyperleucocytose, une augmentation du taux des créatine-kinases (CPK), une acidose métabolique (3).

Des critères diagnostiques ont été proposés par Sternbach en 1991 (1) : ils reposent sur l'anamnèse, les signes cliniques, le diagnostic différentiel (Tableau I).

Le syndrome malin des neuroleptiques est proche sur le plan sémiologique du syndrome sérotoninergique ; la présence de myoclonies serait plus en faveur de ce dernier. Mais aucun examen paraclinique n'est spécifique du syndrome sérotoninergique.

\section{Traitement}

La majorité des cas de gravité modérée évoluent favorablement en 24 à 72 heures après arrêt des traitements en cours et un traitement symptomatique. Le traitement symptomatique des formes modérées peut comporter des benzodiazépines pour contrôler l'hypertonie neuromusculaire, du paracétamol pour abaisser la température et éventuellement un refroidissement externe. L'apparition d'une hyperthermie majeure est un signe de gravité ; le traitement peut nécessiter une assistance respiratoire et une curarisation. Des décès ont été rapportés dans les formes graves. Dans tous les cas, les autres causes d'hyperthermie et de troubles de la conscience doivent être recherchées. Bien qu'un rapprochement avec le syndrome malin des neuroleptiques soit tentant, un médicament comme la bromocriptine proposé dans le traitement du syndrome malin pourrait aggraver un syndrome sérotoninergique (3). Compte tenu de la difficulté éventuelle à différencier les deux situations la plus grande prudence s'impose donc. La parenté du syndrome sérotoninergique avec l'hyperthermie maligne n'est pas très claire non plus ; bien qu'il ne semble pas aggraver le syndrome, la place du dantrolène n'est pas encore bien définie. D'autres traitements ont été utilisés à partir de données issues de l'expérimentation animale ou de cas cliniques isolés : propranolol (affinité spécifique pour le récepteur 5HT1A et correction du syndrome sérotoninergique chez l'animal), méthysergide, cyproheptadine (antagonistes non spécifiques de la sérotonine). En l'absence d'études cliniques contrôlées, il est difficile de recommander leur utilisation $(2,3)$.

\section{Pharmacocinétique des IRS et IRSNA}

Les IRS, et plus récemment les IRSNA (inhibiteur de la recapture de la sérotonine et de la noradrénaline), sont les médicaments le plus fréquemment impliqués dans le SS. Ils présentent des différences pharmacocinétiques importantes résumées dans le Tableau III (11-15).

Après administration orale ils sont en général bien résorbés et largement répartis dans les tissus. Les IRS (à l'exception du citalopram) et la venlafaxine subissent un important effet de premier passage hépatique. Les IRS ont une demi-vie longue de $15 \mathrm{~h}$ pour la fluvoxamine à quelques jours pour la fluoxétine, les IRSNA ont une demi-vie plus courte (5-8h). Ces antidépresseurs sont presque totalement biotransformés à l'exception du milnacipran dont l'excrétion urinaire sous forme inchangée est d'environ $50 \%$ (15). Les métabolites ont une activité variable selon les molécules. La Norfluoxétine et la Norvenlafaxine sont deux métabolites d'activité importante qui doivent être mesurés conjointement à la molécule mère dans le suivi thérapeutique ou toxique ; la Norfluoxétine à une demi-vie particulièrement longue de 4 à 15 jours. La fluoxétine, la fluvoxamine, la paroxétine et la venlafaxine présentent une pharmacocinétique non linéaire (11).

Le métabolisme est hépatique via la voie oxydative du cytochrome $\mathrm{P} 450$ et fait intervenir différents isoenzymes. Ils peuvent inhiber différents cytochromes : CYP2D6, CYP2C19, CYP1 A2 et CYP3A4 (16). Leur potentialité inhibitrice est variable (11-16), les risques les plus importants d'interaction métaboliques sont avec la fluoxétine, la paroxétine et la fluvoxamine. Le citalopram, la sertraline et la venlafaxine ont une affinité plus faible pour les cytochromes et présentent moins de risques d'interactions. Le milnacipran, principalement éliminé par voie rénale, est dénué d'effet inhibiteur métabolique. Des effets inhibiteurs ont été décrits sur le métabolisme des antidépresseurs tricycliques mais également sur ceux d'autres substances dont la théophylline, la clozapine, l'alprazolam, le propranolol $(13,17,18)$.

Les concentrations plasmatiques des IRS et IRSNA sont variables, les concentrations dites «thérapeutiques» du Tableau III sont celles observées pour les doses efficaces recommandées. Ces antidépresseurs ont un index thérapeutique large, ils présentent une importante variabilité pharmacocinétique et pharmacodynamique $(12,13,19)$ et selon les études la relation directe concentration plasmatique/efficacité n'est pas bien établie $(13,20,21,22)$. Le suivi des concentrations plasmatiques est recommandé pour des populations particulières telles que les sujets âgés, les patients présentant des déficiences en cytochrome $\mathrm{P} 450$ ou les insuffisants hépatiques (21-23). Les IRS ou IRSNA, seuls, ont une 
Tableau III : Paramètres pharmacocinétiques des IRS et IRSNA.

\begin{tabular}{|c|c|c|c|c|c|c|c|c|c|c|}
\hline & DCI & SPÉCIALITÉS & \begin{tabular}{|c|}
$\begin{array}{c}\text { Posologie } \\
\mathrm{mg} / \mathrm{j}\end{array}$ \\
\end{tabular} & $\begin{array}{c}\text { Biodisp } \\
\% \\
\end{array}$ & $\begin{array}{c}\text { Liais Prot } \\
\% \\
\end{array}$ & $\begin{array}{c}\mathrm{Vd} \\
\mathrm{L} / \mathrm{Kg}\end{array}$ & $\begin{array}{c}\text { Cmax } \\
\mathrm{h} \\
\end{array}$ & Demi-vie & $\begin{array}{c}\text { C Thérap } \\
\mu \mathrm{g} / \mathrm{L}\end{array}$ & Métab *Actif \\
\hline \multirow[t]{6}{*}{ IRS } & Citalopram & SEROPRAM* & $20-60$ & 80 & 50 & 15 & 4 & $33 \mathrm{~h}$ & $50-200$ & moins actif \\
\hline & Fluoxétine & PROZAC* & $20-60$ & 70 & 94 & $15-40$ & 6 & $2-7 j$ & $150-500$ & Norfluoxétine* \\
\hline & & & & & & & & & $+\mathrm{M}: 250-1000$ & \\
\hline & Fluvoxamine & FLOXYFRAL* & $100-300$ & 50 & 77 & 25 & $2-8$ & $16 \mathrm{~h}$ & $50-250$ & 2 fois - actif \\
\hline & Paroxétine & DEROXAT* & $20-50$ & 50 & 95 & 17 & & $24 \mathrm{~h}$ & $10-75$ & peu actifs \\
\hline & Sertraline & ZOLOFT* & $50-100$ & 90 & 98 & 20 & $5-8$ & $26 \mathrm{~h}$ & $50-250$ & 10 fois - actif \\
\hline \multirow[t]{2}{*}{ IRSNA } & Milnacipran & IXEL* & $100-200$ & 85 & 13 & 5 & 2 & $8 \mathrm{~h}$ & $100-300$ & négligeable \\
\hline & Venlafaxine & EFFEXOR* & $75-150$ & 50 & 27 & 7 & 2,5 & $5 \mathrm{~h}$ & $100-200$ & Norvenlafaxine* \\
\hline
\end{tabular}

faible toxicité et des concentrations toxiques allant de $0,5 \mathrm{mg} / \mathrm{l}$ pour la paroxétine à $3 \mathrm{mg} / \mathrm{l}$ pour la fluvoxamine, ont été relevées dans quelques cas associés le plus souvent à d'autres médicaments $(13,18)$.

\section{Approche analytique}

Les antidépresseurs sérotoninergiques et plus fréquemment leur association à d'autres psychotropes, pouvant être à l'origine du syndrome sérotoninergique, l'approche de toxicologie analytique va consister en l'identification des substances médicamenteuses présentes et/ou de leur dosage dans le suivi de l'intoxication.

La diversité des molécules incriminées, non reconnues par les techniques immunologiques rapides va nécessiter la mise en route de différentes méthodes analytiques, essentiellement chromatographiques dont un «screening» large par chromatographie liquide ou gazeuse avec une sensibilité permettant si possible la détection des taux thérapeutiques. Les méthodes de dosage, spécifiques ou par famille, sont également chromatographiques (sauf pour le lithium et la recherche urinaire des méthamphétamines). Ces méthodes chromatographiques nécessitent une préparation préalable de l'échantillon sanguin ou urinaire.

\section{«Screening» chromatographique}

Il n'existe pas un système chromatographique capable de détecter, par une seule méthode, l'ensemble des médicaments et toxiques. Cependant l'essentiel des substances intervenant dans le SS peuvent être détectées par les méthodes de «screening» chromatographique systématiques des produits basiques et neutres. Elles utilisent aujourd'hui des systèmes de détection spécifique tels que les spectres UV en CLHP et les spectres de masse en CPG ou en chromatographie liquide, si possible sans dérivation dans une première approche de toxicologie hospitalière (24-26).
Les substances recherchées seront extraites, du sang ou des urines, selon des procédés liquide/liquide par différents mélanges de solvants ou liquide/solide $(24,26,27)$, le même extrait pouvant être injecté dans les systèmes CLHP et CPG pour une analyse complémentaire. L'extraction liquide/solide peut être automatisée en ligne (28).

\section{«Screening» chromatographique CLHP-Barrette de Diode}

Méthodes CLHP en phase inverse, la séparation est réalisée sur colonne greffée $\mathrm{C} 18$ ou $\mathrm{C} 8$ avec une phase mobile acide en mode isocratique ou gradient. Les spectres UV des substances éluées peuvent être identifiées automatiquement par leurs caractéristiques spectrales (29-31). Un système automatisé en ligne (REMEDI, Biorad) permet de traiter l'ensemble de la procédure : purification, séparation et identification $(32,33)$.

\section{«Screening» chromatographique CPG-SM}

Séparation sur une colonne apolaire, détection par spectrométrie de masse avec ionisation par impact électronique et acquisition des données en spectre complet. L'identification se faisant par comparaison aux données de banques de spectres. La fragmentation de certains antidépresseurs nécessite une sélection préalable des fragments caractéristiques. La quantification des composés est réalisée sur un ion spécifique associé à la présence et l'intensité relative de un ou deux ions de confirmation et du temps de rétention. Des méthodes automatisées CG-SM incluant l'identification en bibliothèque ont été développées par différents auteurs (34-37).

\section{«Screening» chromatographique CL-SM}

La chromatographie liquide associée à la spectrométrie de masse s'est développée récemment en toxicologie analytique grâce aux interfaces à pression atmosphé- 
rique, elle permet une plus grande gamme de polarité que la CG-SM et plus de sensibilité que la CLHPBarrette de Diodes. Des méthodes de screening se développent (38-40), mais cet équipement, coûteux, est rare dans les laboratoires hospitaliers.

\section{Méthodes de dosage spécifiques des IRS et IRSNA}

Les IRS, molécules basiques de structures chimiques hétérogènes, ont conduit dans un première approche à des méthodes analytiques spécifiques utilisant différents modes de chromatographie et de détection. Ces méthodes spécifiques et sensibles permettent de quantifier la molécule mère et les différents métabolites, elles ont été développées pour les études pharmacocinétiques (41). Le citalopram, la fluoxétine et le milnacipran, introduits en thérapeutique sous la forme de composés racémiques, ont conduit au développement de méthode HPLC chirales pour la mesure de leurs différents énantiomères (13).

Quelques méthodes décrites plus récemment permettent d'identifier et de doser l'ensemble de la famille : molécules mères et métabolites, dans les mêmes conditions analytiques; elles sont proches de celles appliquées aux antidépresseurs tricycliques. Les IRS, IRSNA et leurs métabolites peuvent être extraits par des procédures liquide/solide ou liquide/liquide en milieu alcalin suivi d'une réextraction acide. Ces méthodes utilisent la chromatographie liquide avec différents types de détection : UV (42-43), fluorimétrique $(43,44)$, masse (45), ou la CPG/SM (46). Les limites de quantification varient selon le type de détection, elles seraient de l'ordre de $30 \mathrm{ng} / \mathrm{ml}$ en UV et de $10 \mathrm{ng} / \mathrm{ml}$ en masse.

Une étude multicentrique sur le dosage des IRS et IRSNA en toxicologie hospitalière (CLHP-UV) par Lamiable et col. (43) illustre l'intérêt de procédures analytiques standardisées d'autant qu'il s'agit d'une famille chimiquement hétérogène présentant divers métabolites actifs ou non.

\section{Discussion}

Efficaces et bien tolérés les antidépresseurs inhibiteurs de la recapture de la sérotonine sont une classe très prescrite. L'intoxication par ces nouveaux antidépresseurs, présentant peu d'effets anticholinergiques et cardiotoxiques, est moins sévère que l'intoxication par les tricycliques mais l'association à d'autres médicaments, même à taux thérapeutique, peut être à l'origine de syndrome sérotoninergique pouvant engager le pronostic vital. L'approche de toxicologie analytique consiste d'une part en l'identification des substances médicamenteuses présentes dans un but de confirmation du diagnostique et/ou de leur dosage dans le suivi de l'intoxication.
Les techniques de dépistage rapide des antidépresseurs dans les milieux biologiques sont des méthodes immunochimiques limitées aux seuls anti-dépresseurs tricycliques et présentant des réactions croisées avec certains neuroleptiques. La grande variété structurale des différents antidépresseurs, leur fréquence dans les intoxications médicamenteuses volontaires et leur toxicité seuls ou associés à d'autres psychotropes nous conduit à une approche de toxicologie analytique large et spécifique. Les méthodes de «screening» chromatographique utilisant la CLHP-Barrette de diodes ou la CPG-SM permettent la détection conjointe de différentes classes pharmacologiques avec en général une moindre sensibilité que les méthodes spécifiques de chaque molécule. Les dosages sont également chromatographiques, principalement CLHP-UV ou CPG-SM Des méthodes appliquant les mêmes conditions analytiques à l'ensemble de la famille IRS-IRSNA ont été décrites récemment, elles sont intéressantes dans le suivi des intoxications par l'une ou l'autre de ces molécules.

\section{Références}

1. Sternbach H. The serotonin syndrome. Am. J. Psychiatry $1991 ; 148: 705-713$.

2. Sporer K.A. The serotonin syndrome-implicated drugs, pathphysiology and management. Drug Saf. 1995 ; 13 : 94-104.

3. Martin T.G. Serotonin syndrome. Ann. Emerg. Med. $1996 ; 28: 520-526$.

4. Baubet T. Peronne E. Le syndrome sérotoninergique : revue critique de la littérature. Rev. Méd. Interne. 1997 ; $18: 380-387$.

5. Kolecki P. Isolated venlafaxine-induced serotonin syndrome. J. Emerg. Med. 1997 ; 15 : 491-493.

6. Ohman R., Spigset O. Serotonin syndrome produced by a combination of fluoxetine and lithium. Pharmacopsychiatry. 1993 ; 26 : 263-264.

7. Mekler G., Woggon B. A case of serotonin syndrome caused by venlafaxine and lithium. Pharmacopsychiatry. $1997 ; 30: 272-273$

8. Sobanski T., Bagli M., Laux G., Rao M.L. Serotonin syndrome after lithium add-on medication to paroxetine. Pharmacopsychiatry. 1997 ; 30 : 106-107.

9. Egberts A.C., Ter Borgh J., Brodie-Meijer C.C. Serotonin syndrome attributed to tramadol addition to paroxetine therapy. Int. Clin. Psychopharmacol. 1997 ; 12 : 181-182.

10. Gardner D.M., Lynd L.D. Sumatripans contraindications and the serotonin syndrome. Ann. Pharmacother. 1998 ; 32 : 33-38.

11. Hiemke C., Hartter S.. Pharmacokinetics of selective serotonin reuptake inhibitors. Pharmacol. Ther. 2000 ; 85 : 11-28.

12. Caccia S. Metabolism of the newer antidepressants. An overview of the pharmacological and pharmacokinetic implications. Clin. Pharmacokinet. 1998 ; 34 : 281-302.

13. Baumann P. Pharmacokinetic-pharmacodynamic relationship of the selective serotonin reuptake inhibitors. Clin. Pharmacokinet. $1996 ; 6$ : 444-469. 
14. Ereshefsky L., Dugan D. Review of the pharmacokinetics, pharmacogenetics, and drug interaction potential of antidepressants : focus on venlafaxine. Depress. Anxiety. 2000 ; 12 Suppl $1: 30-44$.

15. Puozzo C., Albin H., Vincon G., Deprez D., Raymon J.M., Amouretti M. Pharmacokinetics of milnacipran in liver impairment. Eur. J. Drug Pharmacokinet. 1998 ; 23 : 273279.

16. Montastruc J.L., Sénard J.M., Lapeyre-Mestre M. Thérapie. 1997 ; 52 : 143-149.

17. Brosen K.. Differences in interactions of SSRIs. Int. Clin. Psychopharmacol. 1998 ; Suppl 5 : S45-47.

18. Goeringer K.E., Raymon L., Christian G.D., Logan B.K.. Postmortem forensic toxicology of selective serotonin reuptake inhibitors : a review of pharmacology and report of 168 cases. J. Forensic Sci. $2000 ; 45: 633-648$.

19. Steimer W., Muller B., Leucht S., Kissling W. Pharmacogenetics : a new diagnostic tool in the management of antidepressive drug therapy. Clin. Chim. Acta. $2001 ; 308: 33-41$.

20. DeVane C.L. Metabolism and pharmacokinetics of serotonin reuptake inhibitors. Cell. Mol. Neurobiol. 1999 ; 19 : 443-466.

21. Rasmussen B.B., Brosen K. Is therapeutic drug monitoring a case for optimizing clinical outcome and avoiding interactions of the selective serotonin reuptake inhibitors ? Ther. Drug Monit. $2000 ; 22$ : 143-154.

22. Lundmark J., Bengtsson F., Nordin C., Reis M., Walinder J. Therapeutic drug monitoring of selective serotonin reuptake inhibitors influences clinical dosing stategies and reduces drug costs in depressed elderly patients. Acta Psychiatr Scand. $2000 ; 101$ : 354-359.

23. Lohmann P.L., Rao M.L., Ludwig M., Griese E.U., Zanger U.M., Morike K., Maier W., Bagli M. Influence of CYP2D6 genotype and medication on the spartein metabolic ratio of psychiatric patients. Eur. J. Clin. Pharmacol. $2001 ; 57: 289-295$.

24. Maurer H.H. Systematic toxicological analysis procedures for acidic drugs and/or metabolites relevant to clinical and forensic toxicology and/or doping control. J. Chromatogr. $1999 ; 733: 3-25$.

25. Dummer O.H. Chromatographic screening techniques in systematic toxicological analysis . J. Chromatogr. 1999 ; $733: 27-45$.

26. Polinetti A. Systematic toxicological analysis of drugs and poisons in biosamples by hyphenated chromatographic and spectroscopc techniques. J. Chromatogr. $1999 ; 733: 47-63$.

27. Sadeg N., Dumontet M. Intérêt de l'extraction en phase solide en toxicologie : exemple d'extraction de 15 substances toxiques et médicamenteuses par 7 colonnes SPE différentes par un protocole unique. Ann. Tox. Anal. 2001 ; vol XIII : 35-40.

28. Chen X.H., Franke J.P., Ensing K., Wijsbeek J., De Zeeuw R.A. Pitfalls and solutions in the development of a fully automated solid-phase extraction method for drug screening purposes in plasma and whole blood. J. Anal. Toxicol. 1993 ; $17: 421-426$.

29. Tacqui A., Kintz P., Mangin P. Systematic toxicological analysis using HPLC/DAD. J. Forensic Sci. 1995 ; 40 : 254-262.

30. Gaillard Y., Pépin G. Use of high-performance liquid chromatography with photodiode-array UV detection for the creation of a 600 -compound library. Application to forensic toxicology. J. Chromatogr. 1997 ; 763 :149-163.
31. Elliot S.P., Hale K.A. Applications of an HPLC-DAD drug screening system based on retention indices and UV spectra. J. Anal. Toxicol. 1998. 22 : 279-289.

32. Binder S.R., Regalia M., Biaggi Mac Eachern M., Mazhar M. Automated liquid chromatographic analysis of drugs in urine by on line sample sample clean up and isocratic multicolumn separation. J. Chromatogr. $1989 ; 473$ :325-341.

33. Kalasinsky K.S., Shaefer T., Binder S.R. Forensic application of an automated drug-profiling system. J. Anal. Toxicol. $1995 ; 19: 412-418$.

34. Smith N.B. Automated identification by computer of the mass spectra of drugs in urine or serum extracts. J. Anal. Toxicol. $1994 ; 18$ : 16-21.

35. Fiers T., Maes V., Sevens C. Automation of toxicological screenings on a Hewlett Packard Chemstation GC-MS system. Clin. Biochem. $1996 ; 29$ : 357-361.

36. Polinetti A. A simple automated procedure for the detection and identification of peaks in gas chromatography-continuous scan mass spectrometry. Application to systematic toxicological analysis of drugs in whole human blood. J. Anal. Toxicol. $1996 ; 20: 579-586$.

37. Amick G.D. Modification of Hewlett-Packard Chemstation (G1034C version C.02.00 and G1701AA version C.02.00 and C.03.00) data analysis program for addition of automatic extracted ion chromatographic groups. J Anal Toxicol, 1998, 22, 66-71.

38. Marquet P., Lachâtre G. Liquid chromatography-mass spectrometry : potential in forensic and clinical toxicology. J. Chromatogr. 1999 ; 733 : 93-118.

39. Fitzgerald R.L., Rivera J.D., Herold D.A. Broad spectrum drug identification directly from urine, using liquid chromatography-tandem mass spectrometry. Clin. Chem. $1999 ; 45: 1224-1234$.

40. Rittner M., Pragst F., Bork W.R., Neumann J. Screening method for seventy psychoactive drugs or drug metabolites in serum based on high-performance liquid chromatography-electrospray ionisation mass spectrometry. J. Anal. Toxicol. $2001 ; 25: 115-124$.

41. Eap C.B., Baumann P. Analytical methods for the quantitative determination of selective serotonine reuptake inhibitors for therapeutic drug monitoring purpose in patients. $\mathrm{J}$. Chromatogr. 1996 ; 686 : 51-63.

42. Kristoffersen L., Bugge A., Ludanes E., Slordal L. Simultaneous determination of citalopram, fluoxetine, paroxetine and their metabolitess in plasma and whole blood by high-performance liquid chromatography with ultraviolet and fluorescence detection. J. Chromatogr. $1999 ; 734: 229-246$.

43. Lamiable D., Capolaghi B., Kergueris M.F., Lavit J., Arditti J., Lacroix C., Moulsma M., Houdret N., Charlier C., Turcan A. Recommandations pour la technique d'extraction des IRS en toxicologie hospitalière IXième Congrès de la SFTA, LA CLUSAZ, Mars 2001.

44. Lucca A., Gentilini G., Lopez-Silva S., Soldarini A. Simultaneous determination of human plasma levels of four selective serotonin reuptake inhibitors by high-performance liquid chromatography. Ther. Drug. Monit. 2000 ; 22 : 271-276.

45. Delsenne F., Gaillard Y. Dosage de nouveaux antidépresseurs par couplage CLHP-SM. Toxicorama. 1998 ; vol X : 251-254.

46. Lacassie E., Ragot S., Rabatel J.F., Gaulier J.M., Marquet P., Lachâtre G. Recherche et dosage de 24 antidépresseurs et métabolites dans le sérum par couplage GC/SM. Toxicorama. 1998 ; vol X : 234-244. 